\title{
Developmental delay and its predictors among children under five years of age with uncomplicated severe acute malnutrition: a cross-sectional study in rural Pakistan
}

Javeria Saleem ${ }^{1}$, Rubeena Zakar ${ }^{1}$, Gul Mehar Javaid Bukhari ${ }^{2}$, Aneela Fatima ${ }^{3}$ and Florian Fischer ${ }^{4,5^{*}}$ (D)

\begin{abstract}
Background: In developing countries, malnutrition in children and developmental delays are two major challenges for public health. To achieve the vision of the Sustainable Development Goals from the broader perspective of child health, early identification of developmental delays and timely intervention are crucial. The aim of this study is to assess the prevalence of suspected developmental delay and their predictors in children under the age of 5 years with uncomplicated severe acute malnutrition in rural areas of Pakistan.
\end{abstract}

Methods: A multicentre cross-sectional study was conducted among 185 children with uncomplicated severe acute malnutrition. We screened children aged 6-59 months for their nutritional status and clinical complications. Children fulfilling the inclusion criteria underwent the Denver Development Screening Tool II (DDST-II). The children's global developmental profile was calculated according to the established protocols of DDST-II, which are based on four important domains of development: personal and social behaviour, language, gross motor adaptive skills and fine motor adaptive skills. A pretested questionnaire was used to collect data on socio-demographic and nutritional factors for assessing predictors of developmental delay, which were analysed using a multivariate logistic regression model.

Results: Out of 177 children with severe acute malnutrition, 69 (38.9\%) had normal global development and 108 (61.1\%) had delayed global development. Significant associations were found between global developmental delay and younger children (6-24 months vs. 25-59 months; $A O R=4.53,95 \% \mathrm{Cl}: 1.56-13.10, p<0.01$ ), children who were not exclusively breastfed ( $\mathrm{AOR}=3.07,95 \% \mathrm{Cl}: 1.24-7.56, p=0.01$ ), and a history of contact with a tuberculosis smear-positive adult (AOR $=2.67,95 \% \mathrm{Cl}: 1.30-5.49, p<0.01)$.

\footnotetext{
* Correspondence: florian.fischer1@charite.de

${ }^{4}$ Institute of Public Health, Charité - Universitätsmedizin Berlin, Berlin, Germany

${ }^{5}$ Institute of Gerontological Health Services and Nursing Research, Ravensburg-Weingarten University of Applied Sciences, Weingarten, Germany Full list of author information is available at the end of the article
}

(c) The Author(s). 2021 Open Access This article is licensed under a Creative Commons Attribution 4.0 International License, which permits use, sharing, adaptation, distribution and reproduction in any medium or format, as long as you give appropriate credit to the original author(s) and the source, provide a link to the Creative Commons licence, and indicate if changes were made. The images or other third party material in this article are included in the article's Creative Commons licence, unless indicated otherwise in a credit line to the material. If material is not included in the article's Creative Commons licence and your intended use is not permitted by statutory regulation or exceeds the permitted use, you will need to obtain permission directly from the copyright holder. To view a copy of this licence, visit http://creativecommons.org/licenses/by/4.0/ The Creative Commons Public Domain Dedication waiver (http://creativecommons.org/publicdomain/zero/1.0/) applies to the data made available in this article, unless otherwise stated in a credit line to the data. 
Conclusion: About two thirds of the study participants showed delayed or unstable global development. Thus, according to DDST-II-established protocols, there is a high prevalence of suspected developmental delay among children under the age of five years with uncomplicated severe acute malnutrition in rural areas of Pakistan. Children in their first 2 years of life were at particularly high risk due to insufficient breastfeeding. This emphasizes the need to provide adequate infrastructure and information to parents for the prevention of developmental delay in remote areas.

Keywords: Developmental delay, Severe acute malnutrition, Wasting, Stunting, Children

\section{Background}

Developmental delay indicates extensive deficits and lack of developmental skills that would be appropriate for children at a particular age. It may be exhibited in different domains, including motor adaptive skills, language or personal and social behaviour [1,2]. Grantham et al. estimated that, globally, just over 200 million children under five are unable to attain their developmental potential due to poverty, malnutrition, inappropriate childcare and/or child abuse. Most of these were from South Asia and sub-Saharan Africa [3].

Severe acute malnutrition (SAM), also known as severe wasting, represents the most critical form of undernutrition. This is further classified into complicated or uncomplicated SAM, based on the presence or absence of medical complications [4]. Children with medically complicated SAM will mostly be treated as inpatients at a medical facility, but children with uncomplicated SAM are advised to be nursed at home with high-calorie, micronutrient-enriched food [5]. SAM is responsible for a large proportion of the global disease burden among children and has a high lethality (10-30\%). Those children who survive have compromised physical and cognitive development, which leads to reduced productivity in adulthood [6-8].

Early childhood, referring to the first 5 years of life, is the fastest and most sensitive period of child growth and brain development. This period is easily influenced by poverty and biological and psychosocial risk factors $[1,9]$. Prior studies have shown strong associations between nutritional status and developmental outcomes in developing countries [10-12]. Malnutrition constrains rapid brain development by adversely affecting its structural and functional capacity, resulting in developmental deficits among the children in all domains $[3,13]$. An unhealthy external environment also has a negative impact on children's ability to learn social and developmental skills [11]. Therefore, several social, biological and psychological factors can contribute to developmental delay in children [1].

Monitoring the health and nutritional status of children under five years of age is important to ensure that they achieve their developmental potential, including both physical and psychological well-being [14]. It is also helpful in achieving the Sustainable Development Goals (SDGs), particularly SDG 3 and SDG 4, from the broader perspective of child health beyond mere survival. Globally, an estimated 19 million children suffer from SAM. Of these, around 1.4 million are from Pakistan [6]. Like other developing countries, Pakistan is struggling with the public health issue of child malnutrition [15]. According to the Pakistan Demographic Survey (2017-18), 37.6\% of children aged under five were stunted, with $17 \%$ severely stunted. Furthermore, $7.1 \%$ were found to be wasted, with $2 \%$ severely wasted; and 23\% were underweight [16]. According to the Multiple Indicator Cluster Survey (MICS), Dera Ghazi Khan had the highest prevalence of stunting (41\%) in Punjab [17].

Although sufficient data is available on the nutritional profile of children aged under five in Pakistan, there is still a scarcity of data on the developmental potential of malnourished children. Moreover, the factors influencing child development delays and the associations between these factors in children with severe acute malnutrition have seldom been examined. Therefore, this study aims to assess the prevalence of developmental delays and their predictors in children under the age of five years with uncomplicated severe acute malnutrition in rural Pakistan.

\section{Methods \\ Study design}

A multicentre cross-sectional study was conducted during a one-year period, from November 2016 to November 2017.

\section{Study area}

The study was conducted at the Outpatient Therapeutic Programme (OTP) Centres in the District of Dera Ghazi (DG) Khan in Southern Punjab, Pakistan. DG Khan has the worst indicators of malnutrition (stunting and wasting) in Punjab. According to MICS 2018, 41\% of children under five were stunted and $7.5 \%$ were wasted in DG Khan [17]. This socioeconomically underprivileged district has the highest prevalence of illiteracy (65\% of women and $38.5 \%$ of men aged 15-49 were illiterate [17]). Overcrowding is 
a problem, and the area is frequently affected by floods $[17,18]$.

Out of a total of 16 OTPs in DG Khan region, four centres were selected on the recommendation of the District Health Office. The reason for recommending these four centres was that they were functioning more actively than the others because appropriate numbers of staff and sufficient therapeutic food were available there. These OTP centres were being used for screening the nutritional status of children and the timely recognition and referral of complicated cases to inpatient facilities. Children with acute and moderate malnutrition without complications were treated at these centres with Ready to Use Therapeutic Food (RUTF) as a community-based treatment. These OTP centres were also used to provide nutritional supplements to pregnant and lactating mothers.

\section{Study population}

Overall, 185 children, aged 6-59 months, were selected to participate in the study on condition of receiving their parents' written informed consent. At enrolment, these children had SAM without complications according to World Health Organization (WHO) criteria (mid-upper arm circumference [MUAC] $<115 \mathrm{~mm}$ and a weight-forheight $[Z$ score $]<-3)$, were clinically well, alert and had a good appetite. Children with SAM and complications were not included in the study on baseline assessment. Complications, as defined by the WHO, were hypoglycaemia, hypothermia (axillary temperature < $35^{\circ} \mathrm{C}$ ), hyperpyrexia (axillary temperature $>39^{\circ} \mathrm{C}$ ), anorexia, severe dehydration, grade three pitting oedema, severe anaemia and acute lower respiratory tract infection [5]. Children who were clinically ill due to any of these symptoms or having poor appetite were not included in the study. Instead, they were referred to an inpatient facility for further evaluation and treatment. Children aged over 59 months or below 6 months were also not included. Parents' refusal to participate in the study and a failure to assess a child's developmental status were also exclusion criteria.

\section{Sample size}

For the sample size calculation, WHO Sample Size 2.0 and the following formula were used:

$$
n=\frac{Z^{2} \cdot P \cdot(1-P)}{d^{2}}
$$

with a level of significance of $95 \%(Z=1.96)$, an expected proportion of wasting $(\mathrm{P})$ of $11 \%$ [17], and an expected error (d) of $5 \%$. This leads to a sample size of 151 , which was increased to a total of 185 to enhance the study's precision, strength and accuracy. The eligibility to participate in the study was assessed among 252 children, of whom 67 were excluded, because either they did not meet the inclusion criteria $(n=52)$, or their parents refused to participate $(n=15)$ (Fig. 1).

\section{Data collection}

Study data were collected in three steps. Firstly, an enrolment form comprising anthropometry measurements and medical records (history and physical examination) was filled in by a qualified medical practitioner at the relevant centre. Secondly, a structured and pretested questionnaire was administered by the same medical practitioner to access data on socio-demographic characteristics (family, income, education etc.) and patterns of food consumption. Thirdly, the Denver Development Screening Tool (DDST-II) was used for the development assessment of children by the paediatrician, who was especially deputed for this purpose.

The gestational age of mothers was taken from antenatal records in cases of hospital delivery, or based on maternal recall if the birth took place at home. The corrected age of children $\leq 24$ months who were born prematurely (before 37 weeks of gestation) was used. Corrected age was calculated by deducting the number of weeks of missed gestation from the present age.

Medical history was obtained for symptoms of acute diarrhoea, high-grade fever, lethargy, coughing, shortness of breath, seizures, neurological deficit and anorexia. All of these symptoms were indications of a child being clinically ill. In physical examination, severe dehydration, palmar pallor (as an indication of anaemia), and severe pitting oedema were assessed in the children $[4,5]$. Their vital signs were assessed for hypothermia/hyperpyrexia and respiratory rate. For the assessment of hypoglycaemia, a heel-prick was done and children with glucose concentrations of $<3$ $\mathrm{mmol} / \mathrm{L}$ based on the Dextrostix reagent strip by Roche were deemed to be hypoglycaemic. An appetite test was conducted by giving the child a small amount of RUTF (Plumpy Nut by Nutriset) to eat. A child who did not consume a minimum of one third of a packet (three teaspoons, $30 \mathrm{~g}$ ) of RUTF after three tries was labelled as having poor appetite [4-6]. These children were not included in the study.

\section{Measurements}

\section{Anthropometric measurements}

Anthropometric measurements were conducted by the medical staff at the participating centres, who were specifically trained in child nutritional assessment by the master trainers from the nutrition programme of the Provincial Health Department. Assessments were made twice: first by a trained Lady Health Worker (LHW) and then by the Medical Officer of the centre to avoid 


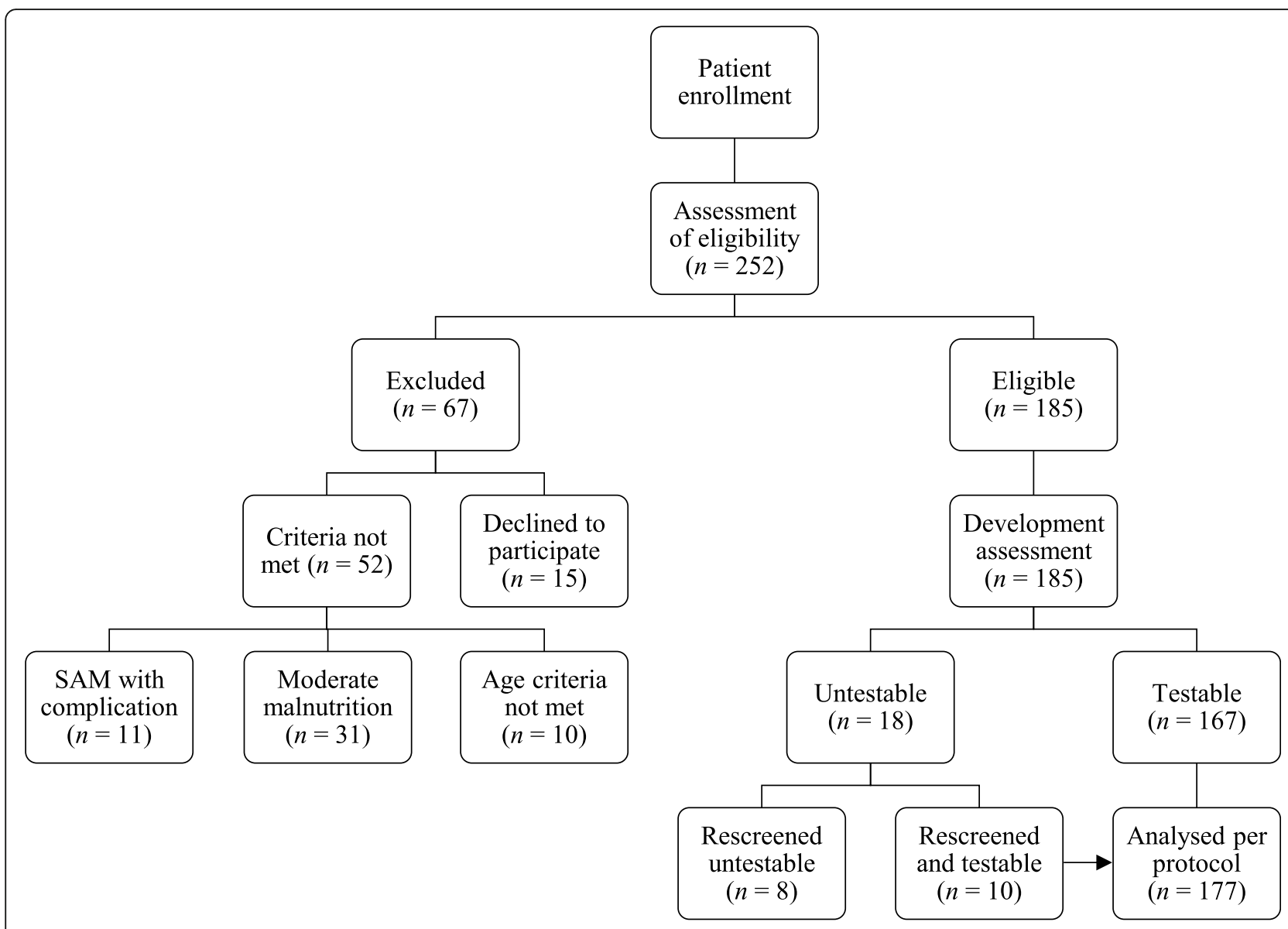

Fig. 1 Flowchart of sample selection and developmental screening

potential bias. In cases where the two measurements differed, further assessments were conducted until a precise value was obtained. The repeated value was then documented. The mid upper arm circumference (MUAC) was assessed to the nearest $0.1 \mathrm{~cm}$ with colour-labelled MUAC tape at the midpoint between the olecranon and the acromion process.

Child weight was measured using UNISCALE (SECA 878), to the nearest $10 \mathrm{~g}$, undressed or in a light dress. If children or infants were not able to stand by themselves, UNISCALE was utilized to determine only the mothers' weight. Afterwards, the infant/child was handed to the mother while she was standing on the scale and the collective infant and mother weight was assessed. The calculation of infant/child weight was determined as the difference between these two readings. UNISCALE was calibrated for standard weight and adjusted to zero prior to all measurements.

The recumbent length of children who were less than or equal to $87 \mathrm{~cm}$ in height was assessed to the nearest $0.1 \mathrm{~cm}$ with the help of a length-measuring board with a fixed headrest and a mobile foot piece ("SECA GmbH \& Co. KG, Hamburg, Germany"), laid on a smooth surface in supine position. Children taller than $87 \mathrm{~cm}$ in height were assessed using Stadiometer (SECA 217) in a standing position after removing shoes and with heels together on a horizontal flat plate attached to the base of the measuring board [18]. Standard protocols for child growth were applied for calculating the weight-forheight Z-score using WHO ANTHRO version 3.2.2.

\section{Developmental screening}

Study participants underwent the DDST-II [19] in order to assess their development profile. This tool measures the proficiency of children under the age of six years to do a range of different tasks and makes comparisons with a standardized population of same-age children. It measures the four parameters of development: personalsocial behaviour development, fine motor development, language, and gross motor development. This tool was applied by a physician trained in conducting DDST-II for child development assessment. The average screening time for DDST-II was about $30 \mathrm{~min}$. If the child was uncooperative and untestable at the first screening for global developmental status, a follow-up rescreen visit was conducted after two days. If the child was still 
untestable, the screening was repeated after a further two days. The tool was translated into the child's native language in order to eliminate communication barriers. The flowchart of developmental screening is presented in Fig. 1.

Following a standardized algorithm, each child's development was categorized as normal, if the child performs the items on the left of the age line completely. There is a further category entitled "caution", which is an intermediate classification, for children who are unsuccessful to an item on which the age line falls on or between the 75th and 90th percentiles. Finally, children are categorized as delayed, if they do not pass an item that $90 \%$ of children in the standardization passed at an earlier age and item on which age line falls completely to the left of the age line [19].

These category measurements were then applied to grade global developmental status as "normal" (no category delayed and no more than one category classified as caution), "suspect" ( $\geq 2$ cautions or $\geq 1$ delay), or "untestable" (based on a specific pattern of refusals) [19].

\section{Statistical analyses}

Data was analysed using SPSS version 23. Simple logistic regression was applied to assess the bivariate relationship through unadjusted Odds Ratios (OR) between the dependent and independent variables. The $p$-value was set at 0.20 in order to include all significant variables as confounding variables in the multivariate analysis [20]. Hence, a multivariate logistic regression was run to measure the dynamics among the potential predictors for delayed development among the children and to present the results in terms of Adjusted Odds Ratios (AOR) with 95\% confidence intervals $(95 \% \mathrm{CI}$ ) and level of significance $(p<0.05)$.
The outcome variable was the children's developmental status as a binary variable (delayed development vs. normal development). Socio-demographic characteristics, medical history and dietary practices were included as independent variables, because they can theoretically affect developmental delay (Fig. 2).

\section{Results \\ Developmental status}

Out of the total 185 children, eight (4.3\%) were untestable for developmental status even after rescreening. Therefore, the analysis is based on 177 children. The results for the prevalence of suspected global development delays based on developmental milestones, in all domains and separated for each domain, are presented in Table 1. According to this, $61.1 \%(n=108)$ showed a suspected delay at the global level. The delay was most pronounced in personal and social behaviour (62.1\%, $n=110)$, whereas for language $70.6 \%(n=125)$ showed normal development.

\section{Socio-demographic characteristics and developmental status}

Comparisons of socio-demographic characteristics between children with normal development and suspected developmental delay are presented in Table 2 . The comparison revealed that child's age $(p<0.01)$, monthly income $(p=0.04)$, mother's education $(p=$ $0.01)$, father's education $(p=0.04)$ and family size were $(p=0.01)$ significantly associated with suspected developmental delay. All other socio-demographic factors showed no significant association with developmental status.

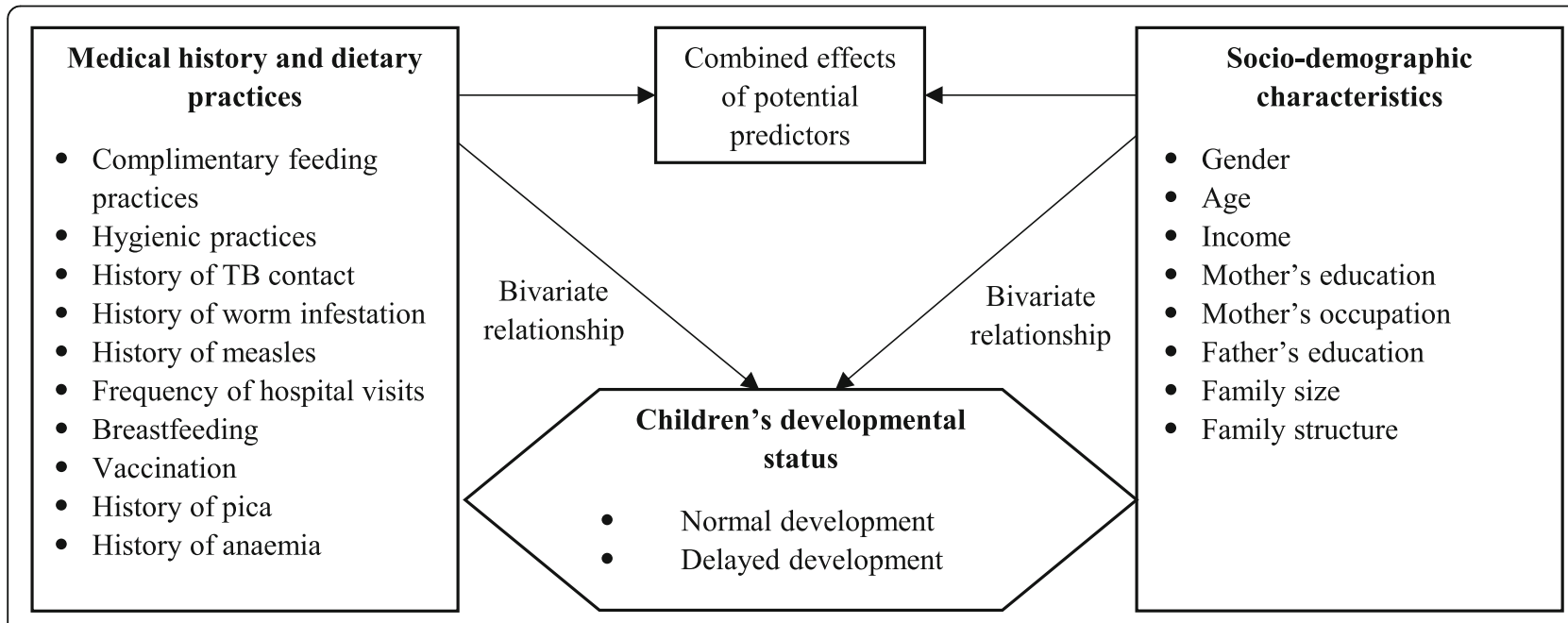

Fig. 2 Relationships between variables affecting developmental delay 
Table 1 Prevalence of suspected global developmental delay $(n=177)$

\begin{tabular}{lll}
\hline & Categories & $\boldsymbol{n}(\%)$ \\
\hline Global development & Normal & $69(38.9)$ \\
Development in domains & Suspected delay & $108(61.1)$ \\
Personal and social behaviour & Normal & \\
& Delay & $67(37.9)$ \\
Fine motor & Caution & $21(45.8)$ \\
& Normal & $29(16.3)$ \\
& Delay & $113(63.8)$ \\
Language & Caution & $23(24.3)$ \\
& Normal & $125(70.6)$ \\
& Delay & $23(13.0)$ \\
Gross motor & Caution & $29(16.4)$ \\
& Normal & $115(65.0)$ \\
& Delay & $28(15.8)$ \\
& Caution & $34(19.2)$ \\
\hline
\end{tabular}

\section{Medical history, dietary practices and developmental status}

A significant association with delayed development was found for the frequency of hospital visits $(p=0.03)$, which are visits to hospital in the past 6 months due to diarrhoea, respiratory infections or because of any other illness (which was confirmed from their medical records to avoid any recall bias); history of tuberculosis (TB) contact $(p=0.01)$ (defined as a child with close contact to a TB smear-positive adult patient at home or in the near surroundings); and breastfeeding practices $(p=0.01)$. Furthermore, a history of worm infestation $(p=0.14)$ was included in the multivariate analysis as the level of significance was set at $p<0.20$ for this analysis. All other variables, such as complementary feeding practices (complementary food was probed in detail from mothers according to WHO recommendations by showing them different utensils and food charts to assess quantity, variety and frequency of feeding), hygiene practices, vaccination, and history of measles, were shown to not be related to delayed development among children (Table 3).

\section{Multivariate logistic regression}

All variables that were found to be significant $(p \leq 0.20)$ in the simple logistic regression models (Tables 2 and 3 ) were included in the multivariate logistic regression analysis. As multicollinearity was found between mother's and father's education, we only kept mother's education in the analysis to account for the fact that mother's education has been found to be a significant predictor of child development in previous studies [21].

In the multivariate logistic regression model, only a younger age $(6-24$ months vs. $25-59$ months) of the child (AOR $=4.53,95 \% \mathrm{CI}: 1.56-13.10, p<0.01)$, history of $\mathrm{TB}$ contact $(\mathrm{AOR}=2.67,95 \% \mathrm{CI}: 1.30-5.49, p<0.01)$, and mixed breastfeeding practices $(\mathrm{AOR}=3.07,95 \% \mathrm{CI}$ :

Table 2 Socio-demographic characteristics and developmental status ( $n=177$ )

\begin{tabular}{|c|c|c|c|c|c|c|}
\hline Socio-demographic characteristics & Categories & $n$ & $\begin{array}{l}\text { Normal development } \\
n(\%)\end{array}$ & $\begin{array}{l}\text { Delayed development } \\
n(\%)\end{array}$ & OR $(95 \% \mathrm{Cl})$ & $p$-value \\
\hline \multirow[t]{2}{*}{ Sex } & Male & 78 & $27(34.6)$ & $51(65.4)$ & $1.39(0.75-2.57)$ & 0.29 \\
\hline & Female & 99 & $42(42.4)$ & $57(57.6)$ & Reference & \\
\hline \multirow[t]{2}{*}{ Age (in months) } & $6-24$ & 151 & $50(33.1)$ & $101(66.9)$ & $5.48(2.16-13.9)$ & $<0.01$ \\
\hline & $25-59$ & 26 & $19(73.1)$ & $7(26.9)$ & Reference & \\
\hline \multirow[t]{2}{*}{ Monthly income (in PKR) } & $<15,000$ & 121 & $41(33.9)$ & $80(66.1)$ & $1.95(1.02-3.72)$ & 0.04 \\
\hline & $\geq 15,000$ & 56 & $28(50.0)$ & $28(50.0)$ & Reference & \\
\hline \multirow[t]{2}{*}{ Mother's education } & Illiterate & 128 & 43 (33.6) & $85(66.4)$ & $2.23(1.14-4.36)$ & 0.01 \\
\hline & $\geq$ Primary & 49 & $26(53.1)$ & $23(46.9)$ & Reference & \\
\hline \multirow[t]{2}{*}{ Mother's occupation } & Working & 19 & $10(52.6)$ & $9(47.4)$ & Reference & 0.20 \\
\hline & Housewife & 158 & $59(37.3)$ & $99(62.7)$ & $1.86(0.72-4.85)$ & \\
\hline \multirow[t]{2}{*}{ Father's education } & Illiterate & 109 & $36(33.0)$ & $73(67.0)$ & $1.91(1.02-3.55)$ & 0.04 \\
\hline & $\geq$ Primary & 68 & $33(48.5)$ & $35(51.5)$ & Reference & \\
\hline \multirow[t]{2}{*}{ Family size } & $<8$ & 40 & $22(55.0)$ & $18(45.0)$ & Reference & 0.01 \\
\hline & $>8$ & 137 & $47(34.3)$ & $90(65.7)$ & $2.34(1.14-4.79)$ & \\
\hline \multirow[t]{2}{*}{ Family structure } & Joint & 72 & $30(41.7)$ & $42(58.3)$ & Reference & 0.54 \\
\hline & Nuclear & 105 & $39(37.1)$ & $66(62.9)$ & $1.21(0.65-2.32)$ & \\
\hline
\end{tabular}


Table 3 Medical history, dietary practices and developmental status $(n=177)$

\begin{tabular}{|c|c|c|c|c|c|c|}
\hline Medical history and dietary practices & Categories & $n$ & $\begin{array}{l}\text { Normal development } \\
n(\%)\end{array}$ & $\begin{array}{l}\text { Delayed development } \\
n(\%)\end{array}$ & OR $(95 \% \mathrm{Cl})$ & $p$-value \\
\hline \multirow[t]{2}{*}{ Complementary feeding practices } & Poor & 136 & $18(43.9)$ & $23(56.1)$ & $1.30(0.64-2.65)$ & 0.46 \\
\hline & Good & 41 & $51(37.5)$ & $85(62.5)$ & Reference & \\
\hline \multirow[t]{2}{*}{ Hygienic practices } & Poor & 157 & $8(40.0)$ & $12(60.0)$ & $1.05(0.41-2.71)$ & 0.92 \\
\hline & Good & 20 & $61(38.9)$ & $96(61.1)$ & Reference & \\
\hline \multirow[t]{2}{*}{ History of TB contact } & Yes & 77 & $22(28.6)$ & $55(71.4)$ & $2.22(1.18-4.17)$ & 0.01 \\
\hline & No & 100 & $47(47.0)$ & $53(53.0)$ & Reference & \\
\hline \multirow[t]{2}{*}{ History of worm infestation } & Yes & 41 & $12(29.3)$ & $29(70.7)$ & $1.74(0.82-3.71)$ & 0.14 \\
\hline & No & 136 & $57(41.9)$ & $79(58.1)$ & Reference & \\
\hline \multirow[t]{2}{*}{ History of measles } & Yes & 26 & $9(34.6)$ & $17(65.4)$ & $1.24(0.52-2.98)$ & 0.62 \\
\hline & No & 151 & $60(39.7)$ & $91(60.3)$ & Reference & \\
\hline \multirow[t]{2}{*}{ Frequency of hospital visits } & $1-7$ & 95 & $44(46.3)$ & $51(53.7)$ & Reference & 0.03 \\
\hline & $8-15$ & 82 & $25(30.5)$ & $57(69.5)$ & $1.96(1.05-3.65)$ & \\
\hline \multirow[t]{2}{*}{ Breastfeeding } & Mixed & 143 & 49 (34.3) & $94(65.7)$ & $2.74(1.27-5.89)$ & 0.01 \\
\hline & Exclusive & 34 & $20(58.8)$ & $14(41.2)$ & Reference & \\
\hline \multirow[t]{2}{*}{ Vaccination } & Incomplete & 45 & $14(31.1)$ & $31(68.9)$ & $1.58(0.77-3.24)$ & 0.21 \\
\hline & Complete & 132 & $55(41.7)$ & $77(58.3)$ & Reference & \\
\hline \multirow[t]{2}{*}{ History of pica } & Yes & 58 & $22(37.9)$ & $36(62.1)$ & $1.06(0.56-2.03)$ & 0.84 \\
\hline & No & 119 & $47(39.5)$ & $72(60.5)$ & Reference & \\
\hline \multirow[t]{2}{*}{ History of anaemia } & Yes & 76 & $31(40.8)$ & $45(59.2)$ & $0.87(0.70-1.61)$ & 0.66 \\
\hline & No & 101 & 38 (37.6) & $63(62.4)$ & Reference & \\
\hline
\end{tabular}

Table 4 Multivariate logistic regression for predictors of developmental delay $(n=177)$

\begin{tabular}{lllr}
\hline Independent variables & Categories & AOR (95\% CI) & p-value \\
\hline Age (in months) & $6-24$ & $4.53(1.56-13.10)$ & $<0.01$ \\
& $25-59$ & Reference & \\
Monthly income (in PKR) & $<15,000$ & $1.43(0.64-3.17)$ & 0.37 \\
& $\geq 15,000$ & Reference & \\
Mother's education & Illiterate & $1.65(0.75-3.62)$ & 0.21 \\
& $\geq$ Primary & Reference & \\
Mother's occupation & Working & Reference & 0.22 \\
Family size & Housewife & $0.50(0.16-1.52)$ & \\
History of TB contact & $<8$ & Reference & 0.14 \\
& $>8$ & $1.87(0.80-4.40)$ & \\
History of worm infestation & Yes & $2.67(1.30-5.49)$ & $<0.01$ \\
& No & Reference & \\
Frequency of hospital visits & No & 2.02 (0.85-4.81) & 0.11 \\
& $8-15$ & Reference & \\
Breastfeeding & Reference & 0.10 \\
& Mixed & 1.87 (0.88-3.97) & \\
& Exclusive & Reference & \\
\hline
\end{tabular}

1.24-7.56, $p=0.01$ ) remained significantly associated with developmental delays (Table 4).

\section{Discussion}

This study revealed a significant prevalence of suspected developmental delays (61.1\%) among children aged under five with severe acute malnutrition. Malnutrition in children is one of the main reasons for developmental delay, which leads to a lifelong adverse impact on health $[10,18]$. Undernutrition affects a child's behaviour and temperament. It is also one of the reasons for low economic productivity in adulthood [18, 22]. Due to being lethargic and apathetic, malnourished children have problems in understanding information and they are less interested in their surroundings than well-nourished children. This results in delayed social interaction skills. Personal and social development during the initial years of life is the main factor responsible for desirable mental health and good performance in adulthood [18, 22]. Therefore, the recognition of personal, social and behavioural problems in young children is very important [23]. Within this study, we found that, in the domain of personal and social behaviour, almost half of the children (45.8\%) had delayed development, with an additional $16.3 \%$ being in the 
caution zone. This result is in line with prevous literature and can, therefore, be generalized to other study settings $[18,22]$.

Children in our study were also found to be affected in language and motor adaptive skills. Previous research has explained that malnourished children also suffer from micronutrient deficiencies, such as calcium and vitamin $\mathrm{D}$, which are important for skeletal muscle function. For that reason, a deficiency in these micronutrients could affect motor skills [6]. Nutritional insufficiencies at an acute stage may damage the cognitive profile and entire auditory system in children, resulting in verbal and written language problems. Previous studies have also reported a significant relationship between being underweight and delays in the development of motor and language skills among children $[24,25]$.

Child development proceeds through a gradual, multifaceted interaction between parental and caregivers' education level, living and working conditions, social circumstances, availability of health facilities, and the physical environment. An unconstructive social or external environment during the early years of life is usually linked to compromised development. Every region or community has its own circumstances. For that reason, locally targeted research and interventions are needed to move forward [23]. In the present study, mother's education, family's monthly income, frequent hospital visits due to repeated illness, and family size were significantly linked to developmental delays in the bivariate analysis. This has also been shown in previous studies $[1,3,10,23]$.

Comparable research in Indonesian slum areas and in the United States have revealed that low maternal education and low family income are strongly associated with delayed development in young children [1, 26, 27]. This is due to the fact that maternal education has a direct effect on proper medical follow-up during the antenatal period, effective family planning, optimal nutrition and child healthcare [18]. The socioeconomic status of a family is one of the determinants of the nutritional status of its children. Children belonging to the lower socio-economic class were poorly nourished in comparison to those in the upper socio-economic class. This is due to food insufficiency and lack of food diversity at the household level. Children aged between one and 2 years were found to be significantly malnourished in comparison to older children [28].

In our study, exclusive breastfeeding was significantly associated with normal child development. A strong correlation between breastfeeding and progressive developmental outcomes in children has been proven previously $[28,29]$. This can be explained by the nutritive value of breast milk and the strong emotional bonding between mother and child. Studies also relate breastfeeding with high-scoring achievements in cognitive tests and in motor and mental development [29, 30]. A cluster-randomized trial conducted interventions for breastfeeding promotion and found significant results for children's long-term outcomes in health and neurological development [31].

The risk of developmental delay was found to be increased during the first 2 years of life. It has also been observed previously that, if an infant suffers from malnutrition during early childhood, the risk of developmental delay is enhanced. Furthermore, this is an indication of serious physical or psycho-social problems. Development during infancy and the toddler period is rapid and easily influenced by environmental and socio-demographic variables $[1,27,29]$.

Effective community-based management of acute malnutrition, including RUTF, has gained a good reputation for treating children with SAM at the centres. Several individual-level factors (such as parental education and low socioeconomic status), facility-level factors (including distance from OTP centres, poor-quality public health infrastructure, untrained community-based healthcare workers, the palatability of RUTF), and community-level factors (such as lack of community awareness and knowledge) have proved to be potential barriers in treating children with SAM [32].

In contrast to other studies, the history of a child's close contact with a TB smear-positive adult patient at home or in near surroundings was found to be significant in the present study. In order to understand this phenomenon, more in-depth research is required. One explanation might be that these children could have had undiagnosed latent or active TB due to malnutrition, low immune status $[2,10]$, or the contact with smearpositive TB patients. Therefore, a complete physical examination along with laboratory investigations is required for the diagnosis of TB in these children.

\section{Strengths and limitations}

As well as the missing information on TB status of the children in this study, another major limitation is the cross-sectional design. Since the developmental assessment was taken at a time when acute malnutrition was already present, our data cannot determine whether the discovered deficits are reversible with therapeutic feeding or more permanent. Monitoring the children's developmental and nutritional status over a longer time period would deliver greater insight due to the dynamic nature of growth and development. The strength of our study is that we have used the DDST-II, which is a validated scale for the developmental assessment of children. Furthermore, this assessment was conducted by well-trained medical staff using established protocols. Moreover, to our knowledge, this is the first study in 
rural areas of Punjab, Pakistan, to investigate the loss of developmental potential in children with SAM.

\section{Conclusion}

Children with severe acute malnutrition have a high prevalence of suspected global developmental delay. Thus, timely identification and proper management of developmental delays in children should be reinforced in order to meet optimal growth and development needs. Developmental delay and malnutrition are two major interrelated public health problems, which hinder the achievement of the SDGs for children in low-resource countries. The health and nutritional status of these children have been adversely affected by poor feeding practices. This has directly influenced the children's physical and mental development. The results of this study will be helpful for policymakers seeking to develop strategies for the prevention and treatment of growth failure and developmental delay in these vulnerable malnourished children.

\section{Abbreviations \\ AOR: Adjusted odds ratios; Cl: Confidence interval; DDST-II: Denver Development Screening Tool II; MICS: Multiple Indicator Cluster Survey; MUAC: Mid-upper arm circumference; OR: Odds ratio; OTP: Outpatient therapeutic programs; PKR: Pakistani Rupees; RUTF: Ready-to-use therapeutic food; SAM: Severe acute malnutrition; SC: Stabilization centre; SDG: Sustainable Development Goal; SPSS: Statistical Package for Social Sciences; TB: Tuberculosis; WHO: World Health Organization; WHZ: Weight- for-height z-score}

\section{Acknowledgements}

We thank the families who took part in this study. We are also grateful to Ms. Mudasir Mustafa (Utah State University, USA) for helping us in the analysis. We are thankful to the staff at the Integrated Reproductive Maternal New-born Child Health \& Nutrition Program (IRMNCH), Dera Ghazi Khan, Pakistan for their support conducting the study.

We acknowledge support from the German Research Foundation (DFG) and the Open Access Publication Fund of Charité - Universitätsmedizin Berlin.

\section{Authors' contributions}

JS, RZ, and GMJB conceptualized the idea and conducted study. JS analysed the study. JS and AF drafted the manuscript. RZ and FF revised it critically for important for intellectual content. All authors have approved the final version.

\section{Funding}

This research received no supporting funds from any funding agency in the public, commercial, or not-for-profit sector. Open Access funding enabled and organized by Projekt DEAL.

\section{Availability of data and materials}

Data is available from corresponding author upon reasonable request.

\section{Declarations}

\section{Ethics approval and consent to participate}

Ethical approval was given by the Ethical Review and Advanced Study Research Board of the University of Punjab, Pakistan (ref no; "9/2352-ACAD") and the District Health Office of the DG Khan. Parents were informed about the study objectives in their local language at the health centres by the study team. Written informed consent of parents, verified by signature or thumb impression, was recorded.
Consent for publication

Not applicable.

\section{Competing interests}

The authors declare that they have no competing interests. FF and RZ serves as Associate Editors for BMC Public Health.

\section{Author details}

'Department of Public Health, Institute of Social and Cultural Studies, University of the Punjab, Lahore, Pakistan. ${ }^{2}$ Department of Community Medicine, Federal Medical and Dental College, Islamabad, Pakistan. ${ }^{3}$ Pakistan Council of Scientific and Industrial Research, Islamabad, Pakistan. Institute of Public Health, Charité - Universitätsmedizin Berlin, Berlin, Germany. ${ }^{5}$ Institute of Gerontological Health Services and Nursing Research,

Ravensburg-Weingarten University of Applied Sciences, Weingarten, Germany.

Received: 20 August 2020 Accepted: 1 July 2021

Published online: 15 July 2021

\section{References}

1. Zhang J, Guo S, Li Y, Wei Q, Zhang C, Wang X, et al. Factors influencing developmental delay among young children in poor rural China: a latent variable approach. BMJ Open. 2018;8(8):e021628. https://doi.org/10.1136/ bmjopen-2018-021628.

2. Gupta N, Kabra M. Approach to the diagnosis of developmental delay - the changing scenario. Indian J Med Res. 2014;139:4-6.

3. Grantham-McGregor S, Cheung YB, Cueto S, Glewwe P, Richter L, Strupp B. International child development steering group. Developmental potential in the first 5 years for children in developing countries. Lancet. 2007;369(9555): 60-70. https://doi.org/10.1016/50140-6736(07)60032-4.

4. World Health Organization. WHO child growth standards and the identification of severe acute malnutrition in infants and children: joint statement by the World Health Organization and the United Nations Children's fund. Geneva: World Health Organization; 2009.

5. World Health Organization. Guideline: updates on the management of severe acute malnutrition in infants and children. Geneva: World Health Organisation; 2013.

6. Saleem J, Zakar R, Zakar MZ, Belay M, Rowe M, Timms PM, et al. High-dose vitamin D3 in the treatment of severe acute malnutrition: a multicenter double-blind randomized controlled trial. Am J Clin Nutr. 2018;107(5):72533. https://doi.org/10.1093/ajcn/nqy027.

7. Lelijveld N, Seal A, Wells JC, Kirkby J, Opondo C, Chimwezi E, et al. Chronic disease outcomes after severe acute malnutrition in Malawian children (ChroSAM): a cohort study. Lancet Glob Health. 2016;4(9):e654-62. https:// doi.org/10.1016/S2214-109X(16)30133-4

8. Galler JR, Bryce C, Waber DP, Zichlin ML, Fitzmaurice GM, Eaglesfield D. Socioeconomic outcomes in adults malnourished in the first year of life: a 40year study. Pediatrics. 2012;130(1):e1-7. https://doi.org/10.1542/peds.2012-0073.

9. National Research Council, Institute of Medicine Committee on Integrating the Science of Early Childhood Development. From neurons to neighbourhoods: The science of early childhood development. Washington, DC: National Academies Press; 2000

10. Chattopadhyay N, Saumitra M. Developmental outcome in children with malnutrition. J Nepal Paediatr Soc. 2016;36(2):170-7. https://doi.org/10.3126/ jnps.v36i2.14619.

11. Lo S, Das P, Horton R. A good start will ensure sustainable future for all. Lancet. 2017;389(10064):8-9. https://doi.org/10.1016/S0140-6736(16)31774-3.

12. Bogale A, Stoecker BJ, Kennedy T, Hubbs-Tait L, Thomas D, Abebe Y, et al. Nutritional status and cognitive performance of mother-child pairs in Sidama, Southern Ethiopia. Matern Child Nutr. 2013;9(2):274-84. https://doi. org/10.1111/j.1740-8709.2011.00345.x

13. Engle PL, Black MM, Behrman JR, De Mello MC, Gertler PJ, Kapiriri L, et al. Strategies to avoid the loss of developmental potential in more than 200 million children in the developing world. Lancet. 2007;369(9557):229-42. https://doi.org/10.1016/S0140-6736(07)60112-3.

14. Grove J, Claeson M, Bryce J, Amouzou A, Boerma T, Waiswa P, et al. Maternal, newborn, and child health and the sustainable development goals - a call for sustained and improved measurement. Lancet. 2015; 386(10003):1511-4. https://doi.org/10.1016/S0140-6736(15)00517-6.

15. UNICEF. National Nutritional Survey 2018, Pakistan. New York: UNICEF; 2019. 
16. National Institute of Population Studies (NIPS) [Pakistan] and ICF. 2019. Pakistan Demographic and Health Survey 2017-18. Islamabad and Rockville: NIPS and ICF.

17. World Bank. Pakistan - Multiple Indicator Cluster Survey 2017-2018 (MICSPUN 2017-2018). Available at: https://microdata.worldbank.org/index.php/ca talog/3559.

18. De Onis M, Onyango AW, Van den Broeck J, Chumlea WC, Martorell R. Measurement and standardization protocols for anthropometry used in the construction of a new international growth reference. Food Nutr Bull. 2004; 25(Suppl. 1):S27-36. https://doi.org/10.1177/15648265040251S105.

19. Frankenburg WK, Dodds J, Archer P, Shapiro H, Bresnick B. The Denver II: a major revision and Restandardization of the Denver developmental screening test. Pediatrics. 1992;89(1):91-7.

20. Bursac Z, Gauss CH, Williams DK, Hosmer DW. Purposeful selection of variables in logistic regression. Source Code Biol Med. 2008;3(1):17. https:// doi.org/10.1186/1751-0473-3-17.

21. Thomas D, Strauss J, Henriques M. How does mother's education affect child height? J Hum Resour. 2016;26(2):183-211. https://doi.org/10.2307/14 5920 .

22. Hamadani JD, Fuchs GJ, Osendarp SJ, Khatun F, Huda SN, GranthamMcGregor SM. Randomized controlled trial of the effect of zinc supplementation on the mental development of Bangladeshi infants. Am J Clin Nutr. 2001;74(3):381-6. https://doi.org/10.1093/ajcn/74.3.381.

23. World Health Organization. Developmental difficulties in early childhood: prevention, early identification, assessment and intervention in low-and middle-income countries: a review. Geneva: World Health Organization; 2012.

24. Jimoh AO, Anyiam JO, Yakubu AM. Relationship between child development and nutritional status of under-five Nigerian children. South African J Clin Nutr. 2018;31(3):50-4. https://doi.org/10.1080/16070658.201 7.1387434 .

25. Hill EL. Non-specific nature of specific language impairment: a review of the literature with regard to concomitant motor impairments. Int J Lang Commun Disord. 2001;36(2):149-71. https://doi.org/10.1080/1368282001001 9874.

26. Gunardi H, Nugraheni RP, Yulman AR, Soedjatmiko S, Sekartini R, Medise BE, et al. Growth and developmental delay risk factors among under-five children in an inner-city slum area. Pediatr Indonesian. 2019;59(5):276-83. https://doi.org/10.14238/pi59.5.2019.276-83.

27. Hurt $\mathrm{H}$, Betancourt LM. Turning one year of age in a low socioeconomic environment: a portrait of disadvantage. J Dev Behav Pediatr. 2017:38(7): 493-500. https://doi.org/10.1097/DBP.0000000000000469.

28. Chaudhary P, Agrawal M. Malnutrition and associated factors among children below five years of age residing in slum area of Jaipur City, Rajasthan, India Asian. J Clin Nutr. 2018;11(1):1-8.

29. Rao MR, Hediger ML, Levine RJ, Naficy AB, Vik T. Effect of breastfeeding on cognitive development of infants born small for gestational age. Acta Paediatr. 2002;91(3):267-74. https://doi.org/10.1111/j.1651-2227.2002.tb0 713.x

30. Yakoob MY, Lo CW. Nutrition (micronutrients) in child growth and development: a systematic review on current evidence, recommendations and opportunities for further research. J Dev Behav Pediatr. 2017;38(8):66579. https://doi.org/10.1097/DBP.0000000000000482.

31. Kramer MS, Aboud F, Mironova E, Vanilovich I, Platt RW, Matush L, et al. Breastfeeding and child cognitive development: new evidence from a large randomized trial. Arch Gen Psychiatry. 2008;65(5):578-84. https://doi.org/10.1 001/archpsyc.65.5.578.

32. Puett $\mathrm{C}$, Guerrero $\mathrm{S}$. Barriers to access for severe acute malnutrition treatment services in Pakistan and Ethiopia: a comparative qualitative analysis. Public Health Nutr. 2015;18(10):1873-82. https://doi.org/10.1017/S13 68980014002444

\section{Publisher's Note}

Springer Nature remains neutral with regard to jurisdictional claims in published maps and institutional affiliations. 